\title{
Polyporus Polysaccharide Regulates Proliferation, Apoptosis and Migration of Bladder Cancer Cells by Polarizing Macrophages to M1 Subtype in Tumor Microenvironment
}

\section{Wenyu Jia}

Guangzhou University of Chinese Medicine

\section{Siwan Luo}

Guangzhou University of Chinese Medicine

\section{Gena Lai}

Guangzhou University of Chinese Medicine

\section{Shiqi Li}

Guangzhou University of Chinese Medicine

\section{Shuai Huo}

Guangzhou University of Chinese Medicine

Meifang Li

Guangzhou University of Chinese Medicine

\section{Xing Zeng ( $\square$ zengxing@gzucm.edu.cn )}

Guangdong Hospital of Traditional Chinese Medicine

\section{Research}

Keywords: Bladder cancer, polyporus polysaccharide, THP-1-derived macrophage, tumor microenvironment, JAK2/NF-kB pathway

Posted Date: June 1st, 2020

DOl: https://doi.org/10.21203/rs.3.rs-31455/v1

License: (c) (i) This work is licensed under a Creative Commons Attribution 4.0 International License. Read Full License 


\section{Abstract}

\section{Background}

Polyporus polysaccharide, an active ingredient of traditional Chinese medicinal Polyporus umbellatus, has multiple biological functions, such as anti-cancer, immune-regulating and hepatoprotective activities. The purpose of this study was to investigate the mechanism of PPS activated macrophages in the treatment of bladder cancer.

\section{Methods}

100ng/mL Phorbol myristate acetate (PMA) was used to induce THP-1 human leukemic cells as a macrophage model. Flow cytometry was used to detect the expression of CD14 and CD68 to verify the establishment of macrophage model. After that, Macrophages derived from THP-1 were treated with different concentrations of PPS $(1,10$ and $100 \mathrm{ug} / \mathrm{mL})$. Flow cytometry and RT-PCR were used to detected the expression of CD16, CD23, CD86, CD40 and interleukin (IL)-IB, INOS, IL-6 mRNA. ELISA was used to test the change of IL-1 $\beta$ in macrophage after the treatment with PPS. The conditioned medium from PPSpolarized macrophages was used to detect the effect of activated macrophages on bladder cancer. MTT assay, 5-ethynyl-2ל-deoxyuridine assay, flow cytometry, Transwell assay, and Western blot analysis were used to detect the effects of polarized macrophages on the viability, proliferation, apoptosis, and migration of bladder cancer cells. Western blot was also used to analysis the change of JAK2/NF-KB pathway protein.

Results

PPS promoted the expression of pro-inflammatory factors, such as IL-I $\beta$ and IL- 6 , and surface molecules CD86, CD16, CD23, and CD40 in macrophages and then polarized macrophages to M1 type. The results demonstrated that activated macrophages inhibited the proliferation of bladder cancer cells, regulated their apoptosis, and inhibited migration and epithelial-mesenchymal transformation (EMT). JAK2/NF-KB pathways were downregulated in the anti-bladder cancer process of activated macrophages.

Conclusion

The findings indicated that PPS inhibited the proliferation and progression of bladder cancer by the polarization of macrophages to M1 type, and the JAK2/NF-KB pathway was involved in the anti-tumor process of bladder cancer.

\section{Background}

Bladder cancer is the leading cause of cancer-related deaths in urinary system. Surgical treatment, chemotherapy, and radiotherapy are the main clinical therapeutic regimens for bladder cancer, but the high recurrence rate $(60 \%-70 \%)$ and mortality are still difficult issues to resolve[1]. Therefore, the 
necessity of exploring prospective methods to improve the therapeutic effect on bladder cancer is emphasized.

The role of the tumor microenvironment (TME) in tumorigenesis and development is further recognized in solid tumors. Immune cells, including T cells, B cells, NK cells, macrophages, dendritic cells, and neutrophils, in the TME [2] can promote the deterioration of tumors, increase the invasiveness of tumors, and evade host immune response and anti-treatment response. However, under certain conditions, they can be involved in host immune surveillance and killing in tumor [3]. Anti-inflammatory type-2 (M2 macrophage) can promote the tumorigenesis, development, metastasis, and drug resistance of solid tumors, including bladder cancer [4-6], while pro-inflammatory type-1 (M1 macrophage) in turn inhibits these effects[7-9]. Increasing the proportion of M1-subtype cells in TME is a promising strategy for cancer treatment $[10,11]$. However, the role of M1-like macrophages polarized by plant polysaccharides in bladder cancer remains unclear.

$P$. umbellatus, belonging to Polyporaceae, has been traditionally used for treatment of edema, acute nephritis and diarrhea (The State Pharmacopoeia Commission of PR China, 2010). In addition, $P$. umbellatus is also used in combination with other drugs to treat tumors, such as the application of Wulingsan(WLS) in bladder cancer[12,13]. Polysaccharide extracted from $P$. umbellatus is the main ingredient of the medicine. Previous studies found that polyporus polysaccharide (PPS) could alleviate the adverse reactions of Bacille Calmette-Guerin $\triangle B C G \triangle$ and improve its efficiency[14], and effectively inhibit the progression of $N$-butyl- $N$-(4-hydroxybutyl)-nitrosamine (BBN)-induced bladder cancer in rats[15]. In the following study, PPS was found to increase the expression level of CD86 and CD40 in bladder cancer tissue of rats [14], and regulated the immune activity of macrophages and promoted them to M1-like macrophages [16]. Therefore, it was speculated that PPS inhibited the progression of bladder cancer by regulating macrophage polarization in tumor macroenvironment. The present study aimed to explore the role of PPS-induced macrophages in bladder cancer cells and its potential molecular mechanism.

\section{Methods}

\section{Materials and chemicals}

P. umbellatus was purchased from Kangmei Pharmaceutical Co., Ltd. (Guangdong, China). The RPMI 1640 medium, fetal bovine serum (FBS), and phosphate-buffered saline (PBS) were procured from Hyclone. Lipopolysaccharide (LPS) was procured from Sigma (MO, USA). Primers for real-time reverse transcriptase-polymerase chain reaction (RT-PCR) and TRIzol were purchased from Invitrogen. Revert Aid First-Strand cDNA Synthesis Kit and FastStart Universal SYBR Green Master (ROX) were purchased from Roche. RIPA and primary antibodies for Western blot analysis, including anti-IKB, anti-phospho-IKB, anti-

NF-KB p65, anti-JAK2, anti-phospho-JAK2, and anti-GAPDH, were purchased from Cell Signaling Technology. Secondary antibodies were obtained from Sigma. The Enhanced Chemiluminescence (ECL) Solution Kit was obtained from Millipore (MA, USA). APC mouse anti-human CD14, APC mouse anti- 
human CD16, APC mouse anti-human CD40, FITC mouse anti-human CD86, FITC mouse anti-human CD68, and FITC mouse anti-human CD23 were purchased from BD Systems (BD Biosciences, USA).

\section{Preparation of PPS}

Crushed $P$. umbellatus was mixed with water in the ratio of $1: 10$. After soaking for $1 \mathrm{~h}$, the mixture was boiled at $100^{\circ} \mathrm{C}$ and refluxed two times, filtered, combined with the filtrate, and concentrated. Then, the solution was added with ethanol to the supernatant to adjust the proportion of ethanol to more than $80 \%$. After filtration, the supernatant was left at $4^{\circ} \mathrm{C}$ overnight, precipitated, and freeze-dried to obtain crude polysaccharide. Then, the crude polysaccharide, chloroform, and n-butanol were mixed in a proportion of 25:4:1 and shaken for $3 \mathrm{~min}$. After static stratification, the organic layer and precipitation were discarded, and this protein removal process was repeated until no white precipitation occurred. The supernatant was collected, concentrated to a proper volume by a rotary evaporator, and then freeze-dried; thus, PPS was obtained.

\section{Culture of THP-1 and differentiation into macrophages}

The THP-1 cells were purchased from ATCC and maintained in the RPMI-1640 medium containing 10\% FBS, $100 \mathrm{UI} / \mathrm{mL}$ penicillin, and $100 \mu \mathrm{g} / \mathrm{mL}$ streptomycin at $37^{\circ} \mathrm{C}$ in a humidified incubator with $5 \% \mathrm{CO}_{2}$. The THP- 1 cells $\left(5 \times 10^{5}\right.$ cells $\left./ \mathrm{mL}\right)$ were exposed to $100 \mathrm{ng} / \mathrm{mL}$ phorbol myristate acetate (PMA) for $48 \mathrm{~h}$ and then cultured for $24 \mathrm{~h}$ in the absence of PMA as a recovery period to differentiate into macrophages. Microscopic photography and flow cytometry were used to evaluate the establishment of a macrophage model. The expression of membrane surface molecules CD14 and CD68 of THP-1 were detected by flow cytometry (BD Biosciences).

\section{Analysis of THP-1 membrane surface molecules by flow cytometry}

Macrophage-like cells were treated with PPS (1, 10, and $100 \mu \mathrm{g} / \mathrm{mL})$ or LPS $1 \mu \mathrm{g} / \mathrm{mL}$ for $72 \mathrm{~h}$ (LPSinduced M1 macrophages in the positive control group). The THP-1 cells were collected in a flow tube and washed with 2-mL of PBS three times. After removing the supernatant, the cells were incubated with corresponding antibodies for $30 \mathrm{~min}$ at $4^{\circ} \mathrm{C}$ and protected from light, following analysis with flow cytometry. The expression levels of CD16, CD23, CD40, and CD86 were detected for evaluating the effects of PPS and LPS on THP-1-derived macrophages.

\section{Detection of target gene mRNA by RT-PCR}


THP-1-derived macrophages were treated with PPS $(1,10$, and $100 \mu \mathrm{g} / \mathrm{mL})$ or LPS $1 \mu \mathrm{g} / \mathrm{mL}$ for $6 \mathrm{~h}$, and then the cells were collected for RNA extraction. Total RNA was extracted from THP-1 using TRIzol reagent and converted into cDNA using the Revert Aid First-Strand cDNA Synthesis Kit. PCR was performed according to the FastStart Universal SYBR Green Master. The genes and corresponding primer sequences (forward and reverse) are listed in Table 1.

Table 1: Primer sequences for PCR amplification.

\begin{tabular}{ll} 
Gene & Primer sequence \\
\hline IL-1 $\beta$ & F: 5'-TGATGGCTTATTACAGTGGCAA-3' \\
\cline { 2 - 2 } & R: 3'-TAGTGGTGGTCGGAGATTCG-5' \\
\hline \multirow{2}{*}{ IL-6 } & F: 5'-TGCCAGCCTGCTGACGAA-3' \\
\cline { 2 - 2 } & R: 3'-AGCTGCGCAGAATGAGATGA-5' \\
\hline SNAL1 & F: 5'-CCTGTCTGCGTGGGTTTT1G-3' \\
\cline { 2 - 2 } & R: 3'-CCAGTGAGTCTGTCAGCCTTTGT-5' \\
\hline TWIST1 & F: 5'-CATTCTCAAGAGGTCGTGCCA-3' \\
\cline { 2 - 2 } & R: 3'-GGTTTTGCAGGCCAGTTTGA-5' \\
\hline GAPDH & F: 5'-GAACGGGAAGCTCACTGG-3' \\
\cline { 2 - 2 } & R: 3'-GCCTGCTTCACCACCTTCT-5'
\end{tabular}

\section{Measurement of expression level of cytokine interleukin-1 $\beta$ by enzyme-linked immunosorbent assay}

After being treated with PPS or LPS, the THP-1 cells were cultured in a fresh medium for $24 \mathrm{~h}$. Cell supernatants were collected and stored at $-80^{\circ} \mathrm{C}$ after centrifugation at $4^{\circ} \mathrm{C}$ and $350 \mathrm{~g}$. The expression levels of cytokines were analyzed using an enzyme-linked immunosorbent assay (ELISA) kit (Abcam). The concentration of the standard curve used to quantify interleukin (IL)-1 $\beta$ was in the range of 0.48-100 $\mathrm{pg} / \mathrm{mL}$. The sample was diluted 20 times before measurement so that the measured absorbance was within the range of the standard curve. The absorption at $450 \mathrm{~nm}$ was measured by enzyme labeling to calculate the concentration of cytokines in the sample.

\section{Preparation of THP-1 cell culture medium and grouping of human bladder cancer cells}


The macrophages were incubated with a complete medium containing different concentrations of PPS $(1,10$, and $100 \mu \mathrm{g} / \mathrm{mL})$ or LPS $1 \mu \mathrm{g} / \mathrm{mL}$ for $72 \mathrm{~h}$, and the medium was refreshed for another $24 \mathrm{~h}$. The cell culture supernatant was collected and stored at $-80^{\circ} \mathrm{C}$ after centrifugation at $4^{\circ} \mathrm{C}$ and $350 \mathrm{~g}$. Human bladder cancer cells T24 and EJ were incubated with the THP-1 culture medium and a fresh medium 1:1 for $24 \mathrm{~h}$ or $48 \mathrm{~h}$, respectively, which were used in the following experiment.

\section{Cell proliferation detection using 3-(4,5-dimethyl-2- thiazolyl)-2,5-diphenyl-2-H-tetrazolium bromide assay and 5-ethynyl-2c-deoxyuridine assay}

The 3-(4,5-dimethyl-2-thiazolyl)-2,5-diphenyl-2-H-tetrazolium bromide (MTT) analysis kit and 5-ethynyl-2לdeoxyuridine (EdU) staining assay were used to assess cell viability and proliferative capacity. T24 and EJ cells $\left(6 \times 10^{3}\right)$ were seeded into a 96 -well plate, and 6 parallel wells were set up for each group. Following a 24-h incubation, the cells were incubated with the collected conditioned medium. Then, $20 \mu \mathrm{L}$ of MTT $(5 \mathrm{mg} / \mathrm{mL})$ was added to each well and incubated at $37^{\circ} \mathrm{C}$ for $4 \mathrm{~h}$. The medium was discarded, and 200- $\mu \mathrm{L}$ of dimethyl sulfoxide was added to each well. The 96 -well plate was gently shaken for 10 min. Then, the absorbance was measured at $490 \mathrm{~nm}$. The Cell-Light EdU Apollo 567 In Vitro Kit (RIBOBIO, Guangzhou, China) was used for EdU assay after the cells $\left(3 \times 10^{3} /\right.$ well) were cultured with the supernatant of macrophages treated with PPS or LPS in a 96-well plate. After Apollo and Hoechst fluorescence staining, photographs were taken under an inverted fluorescence microscope (Nikon, Tokyo, Japan), and the number of proliferating cells was counted.

\section{Apoptosis assay}

The T24 and EJ cells were seeded in six-well plates at a density of $2.5 \times 10^{5}$ cells/well and incubated with the supernatant for $24 \mathrm{~h}$. The cells were collected, washed with PBS, resuspended in $300 \mu \mathrm{L}$ of $1 \times$ banding buffer, mixed with 5- $\mu \mathrm{L}$ of Annexin V-FITC to avoid light, and incubated at room temperature for $15 \mathrm{~min}$. Then, $5 \mu \mathrm{L}$ of PI staining was added and incubated at room temperature for $5 \mathrm{~min}$. Apoptosis was detected using flow cytometry. In addition, apoptosis-associated protein markers were detected using Western blot analysis.

\section{Assessment of protein level using Western blot analysis}

The expression of apoptosis-associated protein marker, EMT-related marker, and JAK2/NF-KB signaling pathway protein was detected using Western blot analysis. The cells were split using RIPA lysate containing inhibitors of proteases and phosphorylated proteases. Subsequently, the protein was collected and quantified by bicinchoninic acid assay. Then, $8 \%-12 \%$ SDS-PAGE was used for protein electrophoresis and then transferred to an Immobilon-P membrane (Millipore). The membranes were 
saturated with a blocking solution ( $5 \%$ bovine serum albumin) for $1.5 \mathrm{~h}$ at room temperature and incubated with a primary antibody overnight at $4^{\circ} \mathrm{C}$. The immunoblot was washed five times with TBST, 5 min each time, and incubated with a secondary antibody for $2 \mathrm{~h}$, which were detected using ECL.

\section{Cell migration assay}

The Transwell migration assay was used to evaluate the metastatic ability of cancer cells. The Transwell

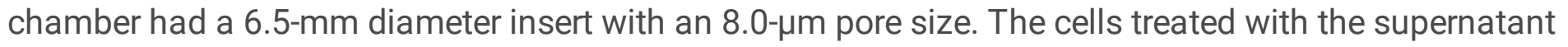
were starved for $2 \mathrm{~h}$ with a serum-free medium. The lower chamber was filled with $600-\mu \mathrm{L}$ of RPMI 1640 medium containing $10 \%$ FBS. The aforementioned treated T24 and EJ cells were counted and resuspended in a serum-free medium, and $200-\mu \mathrm{L}$ of the cell suspension was added to the upper chamber at a density of $2 \times 10^{5} / \mathrm{mL}$. The cells were incubated at $37^{\circ} \mathrm{C}$ for $24 \mathrm{~h}$. The cells penetrating the upper chamber were immobilized with methanol for $30 \mathrm{~min}$ and stained with crystal violet for $20 \mathrm{~min}$. The cells were photographed under a microscope, and the cells passing through the upper chamber were counted.

\section{Statistical analysis}

The data were presented as a mean \pm standard of three independent experimental results. One-way analysis of variance was used for inter-group data. GraphPad Prism 6.0 was used for data processing. A $P$ value $<0.05$ was considered statistically significant.

\section{Results}

\section{Identification of THP-1 differentiation into macrophages}

Macrophage differentiation was closely related to the changes in cell morphology characteristics and cell surface molecules $[17,18]$. Human monocyte THP-1 was incubated with $100 \mathrm{ng} / \mathrm{mL}$ PMA and differentiated into macrophages. Then, the cells became adherent and underwent significant morphological changes. Microscopic photographs showed that PMA-induced cells were enlarged and polygonal, as opposed to the small and round shape of untreated THP-1 cells (Fig 1A). The flow cytometry results showed that, compared with untreated THP-1, the differentiated cells had higher forward scattering (FSC) value and side scattering (SSC) value, reflecting the size of cells and the number of intracellular granules, respectively (Fig 1B). At the same time, the increased expression levels of cell surface molecules CD14 and CD68 in differentiated cells than original THP-1 indicated successful induction of macrophages (Fig 1C-D).

\section{PPS increased the expression levels of inflammatory factors IL-6 and IL-1 $\beta$ and surface molecules CD86, CD16,}




\section{CD23, and CD40 in macrophages}

The polysaccharides extracted from herbal medicines can promote the production of pro-inflammatory factors by macrophages with their good immunoregulatory activity [19-21]. Compared with the control group, PPS increased the mRNA expression levels of IL-6, IL-1 3 , and iNOS (Fig 2A). Furthermore, the ELISA kit was used to detect the change in cytokine levels in the cell supernatant, which demonstrated a significant increase in the expression level of IL-1ß (Fig 2B). CD86 and CD16 were phenotypic molecules produced by M1 macrophages (Smith et al., 2016, Audzevich et al., 2017). The results showed that both PPS and LPS could promote the expression of M1-type membrane molecules CD16, CD23, CD40, and CD86 on the cell surface (Fig 2C).

\section{Macrophage incubated with PPS inhibited the viability and proliferation of human bladder cancer cells}

The MTT assay was used to detect the changes in the viability of T24 and EJ cells incubated with the conditioned medium at different time points. The optical density (OD) values measured in the treatment group showed a downregulated trend compared with the control group. Figure 3A and 3B shows that the cell viability of the experimental group on T24 and EJ cells decreased with the prolongation of culture time. In view of the time-dependent inhibitory effect of the conditioned medium on the viability of bladder cancer cells, the cell proliferation was detected $48 \mathrm{~h}$ later. An EdU nucleoside should always be used to evaluate cell proliferation [24]. Figure 3C and 3D shows that the macrophages activated by PPS attenuated the hyperplasia of T24 and EJ cells in a concentration-dependent manner.

\section{Apoptosis of bladder cancer was enhanced by the PPS- polarized macrophages}

The M1-like macrophages induce apoptosis in cancer cells[25]. Our results showed that PPS-polarized macrophages increased apoptosis obviously compared with the control group, as shown in Figure 5A and 5B. Especially, activated macrophages significantly increased the apoptosis of bladder cancer cells in the late stage rather than in the early stage (Fig. 5C). Apoptosis is characterized by nucleocytoplasmic collapse and cell volume reduction. Flow cytometry showed that the cell volume in the experimental group decreased significantly in the present study (Fig. 5D). Apoptosis-related proteins Bcl-2 and Bax were analyzed by Western blot analysis. Consistent with the result of apoptotic determination by flow cytometry, the expression level of apoptosis inhibitor protein Bcl-2 decreased in the experimental group, while the expression level of apoptosis inducer Bax remained unchanged.

\section{Cell migration and epithelial-mesenchymal transformation ability of bladder cancer cells were regulated}


The ability of cell migration is closely related to the metastasis of bladder cancer. Figure 6A-B shows that the number of cells penetrating the upper chamber decreased significantly after being treated with the cell supernatant of PPS-polarized macrophages, demonstrating that the migration capacity of T24 and EJ cells was inhibited. A close correlation exists between epithelial-mesenchymal transformation and metastasis and invasion of tumors [26]. A conditioned medium of macrophages attenuated the cell migration of bladder cancer cells. Then, the effect of the medium on EMT of bladder cancer was investigated. The expression levels of mesenchymal marker/transcription factors N-cadherin, Snail, Twist, and vimentin in the experimental group significantly decreased, while that of epithelial marker E-cadherin was reversed (Figure 6C-D).

\section{JAK2/NF-KB signaling pathway was involved in the inhibitory effect of PPS-polarized macrophages on bladder cancer cells}

The JAK2 receptor was reported to be closely related to a series of cell functions, such as cell cycle, apoptosis, genetic stability, and tumor cell migration $[27,28]$. The expression of the JAK2/NF-KB signaling pathway protein was examined to explore the potential molecular mechanism of M1-like macrophages regulating the proliferation, apoptosis, migration, and epithelial-mesenchymal transformation of T24 and EJ cells. The Western blot analysis showed that the level of phospho-JAK2 and phospho-NF-KB p65 in T24 and EJ cells decreased after incubation with the conditioned medium (Figure 7). These data indicated that PPS-induced macrophages regulated the proliferation, apoptosis, migration, and epithelial-mesenchymal transformation of bladder cancer cells through the JAK2/NF-KB pathway.

\section{Discussion}

The relationship between tumor cells and immune cells in TME has become a hot topic in recent years. A large number of clinical and experimental data have confirmed that TAMs can promote the genesis and progression of tumors [5]. In view of the role of macrophages in tumors, several anti-cancer strategies targeting macrophages are available[10]. This study demonstrated that PPS played an anti-bladder cancer role by polarizing macrophage to M1 type. Moreover, it also provided evidence that the downregulation of JAK2/NF-KB pathways was involved in the anti-tumor effect of polarized macrophages.

The M1 macrophages produce pro-inflammatory factors (TNF-a, IL-12, and IFN-y), chemokines (CXCL10, CXCL11, and CCL2), antigen-presenting molecules such as MHCII, costimulatory molecules (CD86 and CD80), and antigen-treated peptidases, which play an anti-tumor role in cancer. The M2 macrophages produce nutritional polyamines, anti-inflammatory factors (IL-10 and TGF- $\beta$ ), and chemokines (CXCL18 and CXCL22), which play a tumorigenic role in cancer[29,30]. Therefore, seeking drugs that can induce macrophages to become M1 macrophages and then play an anti-tumor role is of great significance. 
Plant polysaccharides can induce macrophages to polarize to pro-inflammatory subtypes due to their good immunomodulatory effects [31], indicating that polysaccharides regulate TME and have anti-tumor activity. The aqueous extract of $P$. umbellatus Fries was found to effectively inhibit bladder cancer, and polysaccharides were the main component responsible for its effect; however, little is known about its anti-bladder cancer mechanism. The follow-up studies showed that PPS enhanced the activity of macrophages stimulated by IFN-y [16]. In addition, PPS had an inhibitory effect on the progression of bladder cancer in BBN-rat models and increased the effect on the expression levels of CD86 and CD40 in peritoneal macrophages [14]. Therefore, it was speculated that PPS might play an anti-tumor role by regulating the changes in macrophages in the TME of bladder cancer. In this study, in vitro experiments were conducted by simulating the effect of macrophages on tumor cells in TME. PMA-induced THP-1 is a commonly used human macrophage model, which was used in this study. The increased expression levels of CD14 and CD68 on the cell membrane of THP-1 suggested successful modeling, accompanied by increased cell volume. Subsequently, the present study showed that PPS could promote macrophages to secrete inflammatory factors, such as IL-1 $\beta$ and IL-6; increase the expression levels of membrane surface molecules CD86, CD16, CD23, and CD40; and induce macrophages to differentiate into M1 subtype. The anti-tumor effect of M1 macrophages was confirmed. LPS is the classical inducer of M1subtype macrophages, which were used as a positive control in this study. IL-1 $\beta$ and TNF- $a$ have antitumor effects and can inhibit the growth and metastasis of tumors in vitro and in vivo [32,33]. CD40 and CD86 were costimulatory molecules on the surface of macrophages. The increased expression levels of CD40 and CD86 can promote T-cell activation and play an anti-tumor role [34]. Next, the conditioned medium of THP-1 was used in this study to explore the effects of PPS-polarized macrophages on bladder cancer cells. As expected, PPS-induced M1-like macrophages inhibited many pathophysiological characteristics of bladder cancer, including proliferation, migration ability, and epithelial-mesenchymal transformation accompanied by increased apoptosis of bladder cancer cells. At the same time, the sensitivity of T24 and EJ cells to activated macrophages was found to be different in this study, which might be caused by different genotypes and cell receptors. Furthermore, the underlying molecular mechanism of its anti-tumor activity was clarified in the present study. Abnormal activation of JAK2 and $\mathrm{NF}-\mathrm{KB}$ pathways is known to associate inflammation with tumors and is closely related to the malignancy and poor prognosis of tumors [35-37]. The downregulation of JAK2 and NF-KB pathways can regulate the proliferation, apoptosis, and metastasis of tumors[38,39]. In this study, the downregulation of the JAK2/NF-KB pathway was found to be closely related to the anti-cancer effects of PPS-induced polarized macrophages on bladder cancer.

\section{Conclusion}

These findings indicated that PPS could inhibit the growth and progression of bladder cancer cells by polarizing THP-1-derived macrophages to M1 type. The downregulation of JAK2/NF-KB pathways might mediate the anti-cancer process.

\section{Abbreviations}


$P$. umbellatus: Polyporus umbellatus

PPS: polyporus polysaccharide

IL-1 $\beta$ : interleukin (IL)-I $\beta$

IL-6: interleukin (IL)-6

PMA: Phorbol myristate acetate

TNF-a: tumor necrosis factor alpha

MTT: 3-(4,5-dimethyl-2-thiazolyl)-2,5-diphenyl-2-H-tetrazolium bromide

EDU: 5-ethynyl-2c-deoxyuridine

EMT: epithelial-mesenchymal transformation

\section{Declaration}

\section{Ethics approval and consent to participate}

Not applicable.

\section{Consent for publication}

Not applicable.

\section{Availability of data and materials}

The datasets used in the current study are available from the corresponding author on reasonable request.

\section{Competing Interests}

All authors claim no conflict of interests.

\section{Funding}

This study was supported by the National Natural Science Foundation of China (No.81573769) and Special Support for Scientific and Technological Research of Guangdong Provincial Hospital of Traditional Chinese Medicine (No. YN2019MJ02).

\section{Authors' contributions}

Xing Zeng and Wenyu Jia conceived and designed the experiments. Wenyu Jia, Gena Lai, Shiqi Li and Siwan Luo performed the experiments and analyzed the data. Wenyu Jia, Meifang Li and Shuai Huo were 
responsible for the writing and revision of manscript.

\section{Acknowledgements}

Not applicable.

\section{References}

$1 \quad$ Berdik C: Unlocking bladder cancer. NATURE 2017;551:S34-S35.

2 Diakos CIP, Charles KAP, McMillan DCP, Clarke SJP: Cancer-related inflammation and treatment effectiveness. Lancet Oncology, The 2014;15:e493-e503.

3 Klemm F, Joyce JA: Microenvironmental regulation of therapeutic response in cancer. TRENDS CELL BIOL 2015;25:198-213.

4 Giurisato E, Xu Q, Lonardi S, Telfer B, Russo I, Pearson A, Finegan KG, Wang W, Wang J, Gray NS, Vermi W, Xia Z, Tournier C: Myeloid ERK5 deficiency suppresses tumor growth by blocking protumor macrophage polarization via STAT3 inhibition. Proceedings of the National Academy of Sciences 2018;115:E2801-E2810.

5 Qian B, Pollard JW: Macrophage diversity enhances tumor progression and metastasis. CELL 2010;141:39-51.

6 Chen XW, Yu TJ, Zhang J, Li Y, Chen HL, Yang GF, Yu W, Liu YZ, Liu XX, Duan CF, Tang HL, Qiu M, Wang $\mathrm{CL}$, Zheng $\mathrm{H}$, Yue J, Guo AM, Yang J: CYP4A in tumor-associated macrophages promotes premetastatic niche formation and metastasis. ONCOGENE 2017;36:5045-5057.

7 Genin M, Clement F, Fattaccioli A, Raes M, Michiels C: M1 and M2 macrophages derived from THP-1 cells differentially modulate the response of cancer cells to etoposide. BMC CANCER 2015;15:577.

8 Baer C, Squadrito ML, Laoui D, Thompson D, Hansen SK, Kiialainen A, Hoves S, Ries CH, Ooi C, De Palma M: Suppression of microRNA activity amplifies IFN- $\gamma$-induced macrophage activation and promotes anti-tumour immunity. NAT CELL BIOL 2016;18:790-802.

9 Guerra AD, Yeung OWH, Qi X, Kao WJ, Man K: The Anti-Tumor Effects of M1 MacrophageLoaded Poly (ethylene glycol) and Gelatin-Based Hydrogels on Hepatocellular Carcinoma. THERANOSTICS 2017;7:3732-3744.

10 Rubio C, Munera-Maravilla E, Lodewijk I, Suarez-Cabrera C, Karaivanova V, Ruiz-Palomares R, Paramio JM, Dueñas M: Macrophage polarization as a novel weapon in conditioning tumor microenvironment for bladder cancer: Can we turn demons into gods? Clinical and Translational Oncology 2019;21:391-403. 
11 Aldawsari HM, Gorain B, Alhakamy NA, Md S: Role of therapeutic agents on repolarisation of tumour-associated macrophage to halt lung cancer progression. J DRUG TARGET 2020;28:166-175.

12 Xiao Z, Zhang S: Experience of LIN Li -zhu Professor for Bladder Cancer. Journal of Practical Traditional Chinese Internal Medicine 2011;025:16-17.

13 An M, Tao Z, Wu Y: A case study on the treatment of tumor complications with classical prescriptions. Jiangsu Journal of Traditional Chinese Medicine 2014;46:54-55.

14 Zhang G, Qin G, Han B, Li C, Yang H, Nie P, Zeng X: Efficacy of Zhuling polyporus polysaccharide with BCG to inhibit bladder carcinoma. CARBOHYD POLYM 2015;118:30-35.

15 Zhang G, Zeng X, Li C, Li J, Huang Y, Han L, Wei J, Huang H: Inhibition of urinary bladder carcinogenesis by aqueous extract of sclerotia ofPolyporus umbellatus fries and polyporus polysaccharide. The American Journal of Chinese Medicine 2012;39:135-144.

16 Liu C, Zhang X, Tan Q, Xu W, Zhou C, Luo M, Li X, Huang R, Zeng X: NF-kB pathways are involved in $\mathrm{M} 1$ polarization of RAW 264.7 macrophage by polyporus polysaccharide in the tumor microenvironment. PLOS ONE 2017;12:e188317.

17 Shiratori H, Feinweber C, Luckhardt S, Linke B, Resch E, Geisslinger G, Weigert A, Parnham MJ: THP-1 and human peripheral blood mononuclear cell-derived macrophages differ in their capacity to polarize in vitro. MOL IMMUNOL 2017;88:58-68.

18 Daigneault M PJAM: The identification of markers of macrophage differentiation in PMAStimulated THP-1 cells and Monocyte-Derived macrophages. PLoS ONE, 2010

19 Zhang M, Wu W, Ren Y, Li X, Tang Y, Min T, Lai F, Wu H: Structural characterization of a novel polysaccharide fromLepidium meyenii (Maca) and analysis of its regulatory function in macrophage polarization in vitro. J AGR FOOD CHEM 2017;65:1146-1157.

20 Wang M, Yang X, Zhao J, Lu C, Zhu W: Structural characterization and macrophage immunomodulatory activity of a novel polysaccharide from Smilax glabra Roxb. CARBOHYD POLYM 2017;156:390-402.

21 Wang Y, Tian Y, Shao J, Shu X, Jia J, Ren X, Guan Y: Macrophage immunomodulatory activity of the polysaccharide isolated from Collybia radicata mushroom. INT J BIOL MACROMOL 2018;108:300306.

22 Smith TD, Tse MJ, Read EL, Liu WF: Regulation of macrophage polarization and plasticity by complex activation signals. Integrative biology : quantitative biosciences from nano to macro 2016;8:946955. 
23 Audzevich T, Bashford-Rogers R, Mabbott NA, Frampton D, Freeman TC, Potocnik A, Kellam P, Gilroy DW: Pre/pro-B cells generate macrophage populations during homeostasis and inflammation. Proceedings of the National Academy of Sciences 2017;114:E3954-E3963.

24 Diermeier-Daucher S CSTH: Cell type specific applicability of 5-Ethynyl-2 '-deoxyuridine (EdU) for dynamic proliferation assessment in flow cytometry., 2009.

25 Yin Z, Ma T, Lin Y, Lu X, Zhang C, Chen S, Jian Z: IL-6/STAT3 pathway intermediates M1/M2 macrophage polarization during the development of hepatocellular carcinoma. J CELL BIOCHEM 2018;119:9419-9432.

26 Tan TZ, Miow QH, Miki Y, Noda T, Mori S, Huang RYJ, Thiery JP: Epithelial-mesenchymal transition spectrum quantification and its efficacy in deciphering survival and drug responses of cancer patients. EMBO MOL MED 2014;6:1279-1293.

27 Qian C, Yao J, Si J: Nuclear JAK2: Form and function in cancer. The Anatomical Record: Advances in Integrative Anatomy and Evolutionary Biology 2011;294:1446-1459.

28 Wang S, Liang K, Hu Q, Li P, Song J, Yang Y, Yao J, Mangala LS, Li C, Yang W, Park PK, Hawke DH, Zhou J, Zhou Y, Xia W, Hung M, Marks JR, Gallick GE, Lopez-Berestein G, Flores ER, Sood AK, Huang S, Yu D, Yang L, Lin C: JAK2-binding long noncoding RNA promotes breast cancer brain metastasis. J CLIN INVEST 2017;127:4498-4515.

29 Rhee I: Diverse macrophages polarization in tumor microenvironment. ARCH PHARM RES 2016;39:1588-1596.

30 Mills CD, Ley K: M1 and m2 macrophages: The chicken and the egg of immunity. J INNATE IMMUN 2014;6:716-726.

31 Yu Y, Shen M, Song Q, Xie J: Biological activities and pharmaceutical applications of polysaccharide from natural resources: A review. CARBOHYD POLYM 2018;183:91-101.

32 Shen J, Xiao Z, Zhao Q, Li M, Wu X, Zhang L, Hu W, Cho CH: Anti-cancer therapy with TNFa and IFNY: A comprehensive review. CELL PROLIFERAT 2018;51:e12441.

33 Stölting MNL, Ferrari S, Handschin C, Becskei A, Provenzano M, Sulser T, Eberli D: Myoblasts inhibit prostate cancer growth by paracrine secretion of tumor necrosis factor-a. Journal of Urology, The 2013;189:1952-1959.

34 Haabeth O, Blake TR, McKinlay CJ, Tveita AA, Sallets A, Waymouth RM, Wender PA, Levy R: Local delivery of ox40I, cd80, and cd86 mRNA kindles global anticancer immunity. CANCER RES 2019;79:16241634. 
35 Yang C, Zhang W, Wang L, Kazobinka G, Han X, Li B, Hou T: Musashi-2 promotes migration and invasion in bladder cancer via activation of the JAK2/STAT3 pathway. LAB INVEST 2016;96:950-958.

36 Zhang Q, Lenardo MJ, Baltimore D: 30 years of NF-KB: A blossoming of relevance to human pathobiology. CELL 2017;168:37-57.

37 Fan Y, Mao R, Yang J: NF-kB and STAT3 signaling pathways collaboratively link inflammation to cancer. PROTEIN CELL 2013;4:176-185.

38 Zhou Q, Jin P, Liu J, Wang F, Xi S: HER2 and Src co-regulate proliferation, migration and transformation by downstream signaling pathways in arsenite-treated human uroepithelial cells. METALLOMICS 2018;10:1141-1159.

39 Liu B, Sun L, Liu Q, Gong C, Yao Y, Lv X, Lin L, Yao H, Su F, Li D, Zeng M, Song E: A cytoplasmic NF-kappaB interacting long noncoding RNA blocks lkappaB phosphorylation and suppresses breast cancer metastasis. CANCER CELL 2015;27:370-381.

\section{Figures}



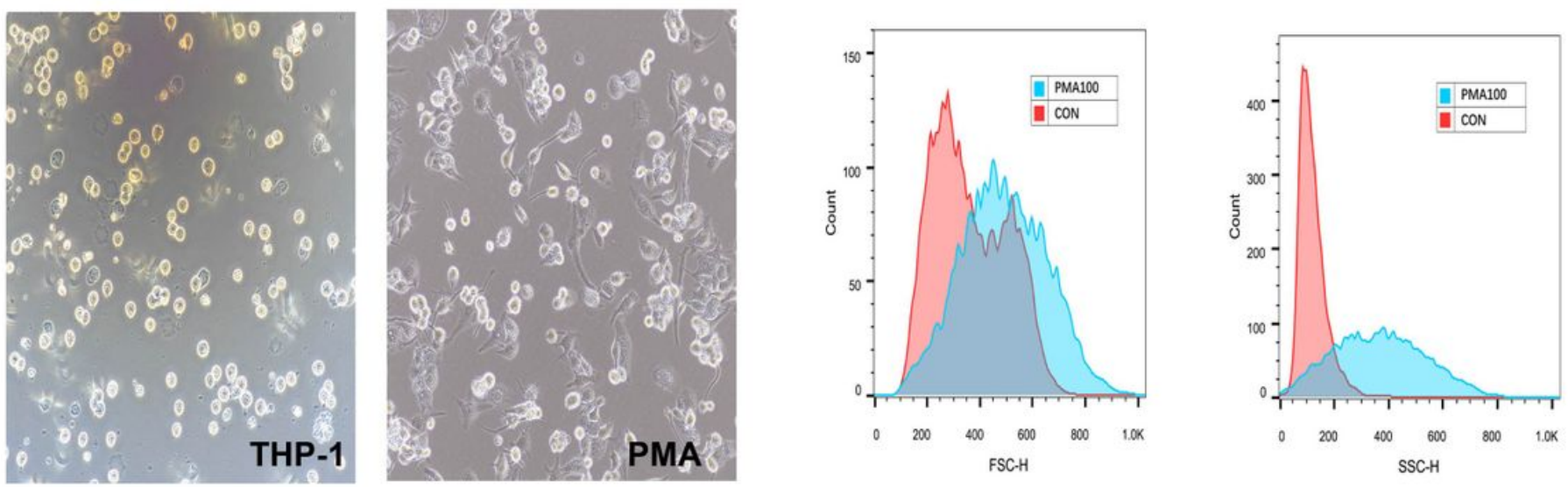

A
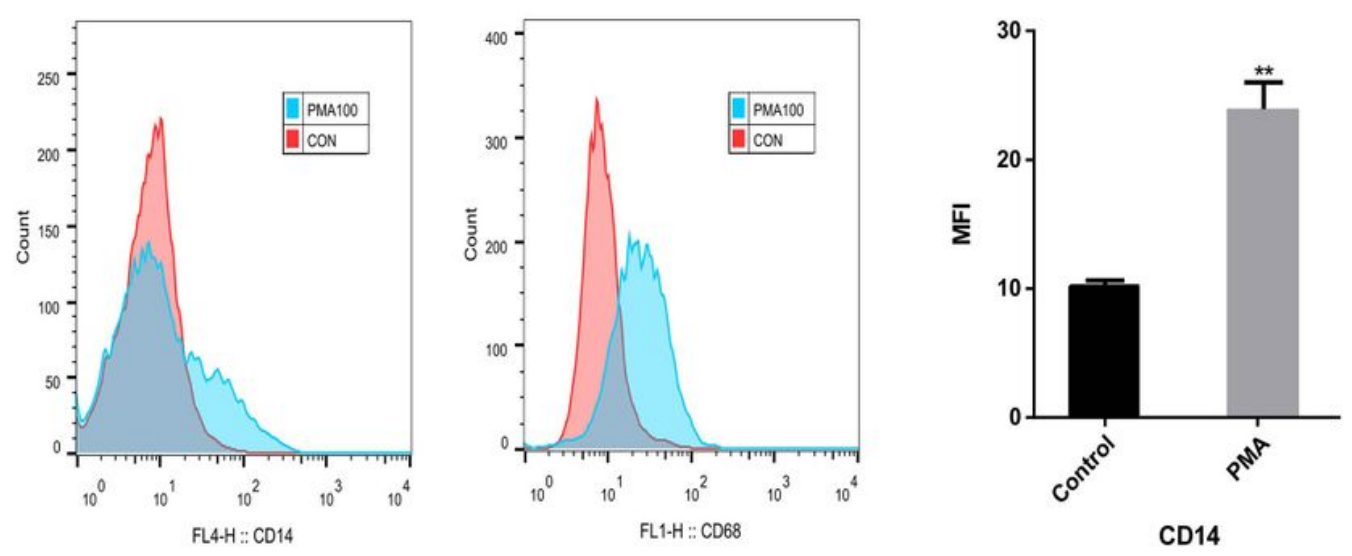

B

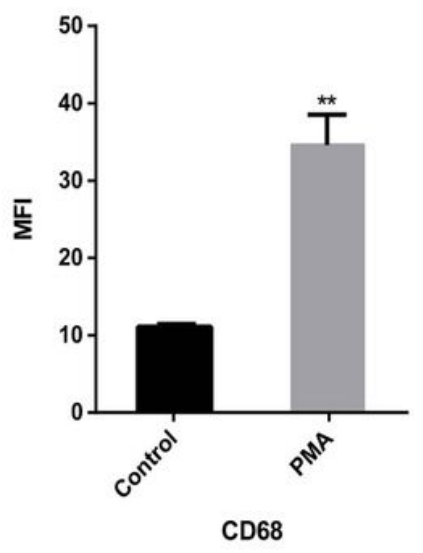

C

D

\section{Figure 1}

THP-1 differentiated into macrophages successfully. (A) Cell morphology observed under a microscope (200x), THP-1 (left), and PMA-induced macrophages (right). (B) Changes in FSC and SSC during flow cytometry before and after cell differentiation. (C) Expression levels of cell surface molecules CD14 and CD68 of THP-1 increased after incubation with PMA. (D) Mean fluorescence intensity of CD14 and CD68 before and after differentiation. ${ }^{*} P<0.05 ;{ }^{*} \mathrm{P}<0.01$ compared with the control group $(\mathrm{n}=3)$. 

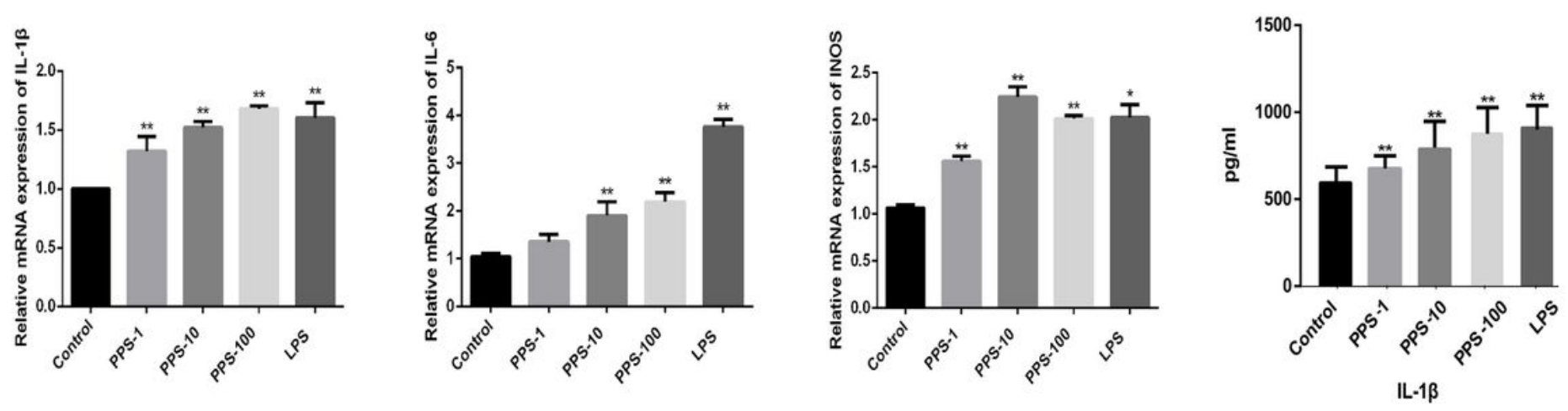

A
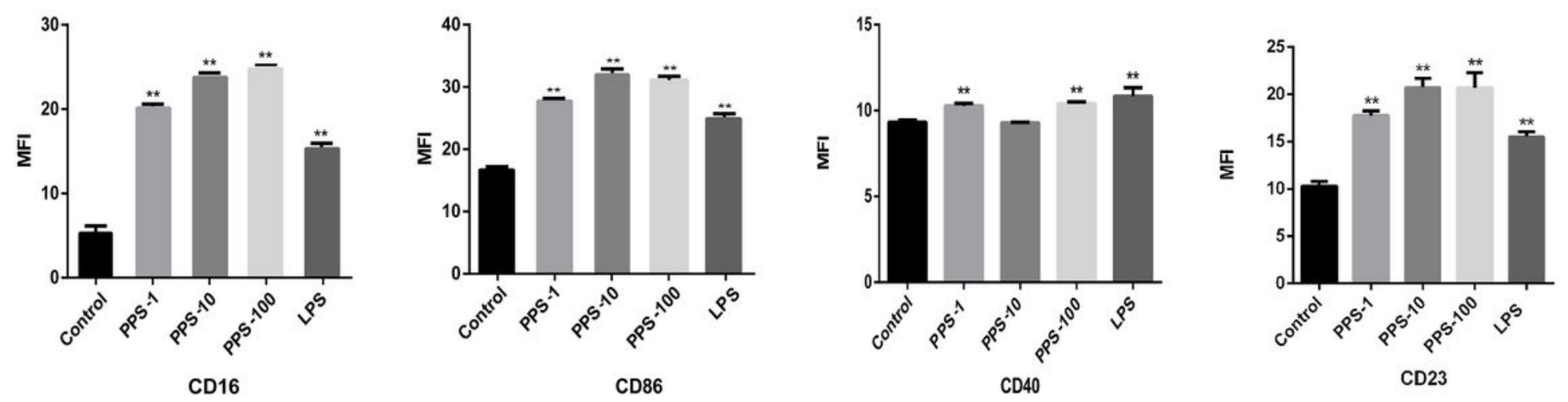

C

\section{Figure 2}

PPS increased the expression levels of inflammatory cytokines, such as IL-1ß, IL-6, and NO, secreted by macrophages and promoted the expression of surface molecules CD86 and CD16. (A) mRNA expression of IL-6, IL-1 $\beta$, and iNOS in macrophages after $6 \mathrm{~h}$ of PPS or LPS incubation. (B) PPS or LPS induced IL-1 $\beta$ production in the cell supernatant and the expression levels cytokines were analyzed by ELISA. (C) THP-1derived macrophages were pretreated with PPS or LPS for $72 \mathrm{~h}$, then the expression levels of CD23, CD40, CD16, and CD86 were measured by flow cytometry. ${ }^{*} P<0.05 ; * \star P<0.01$ compared with the control group $(n=3)$. 


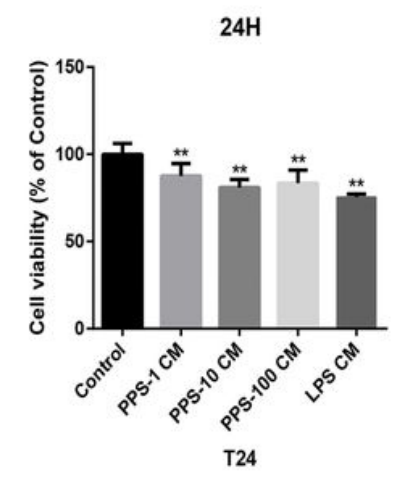

A

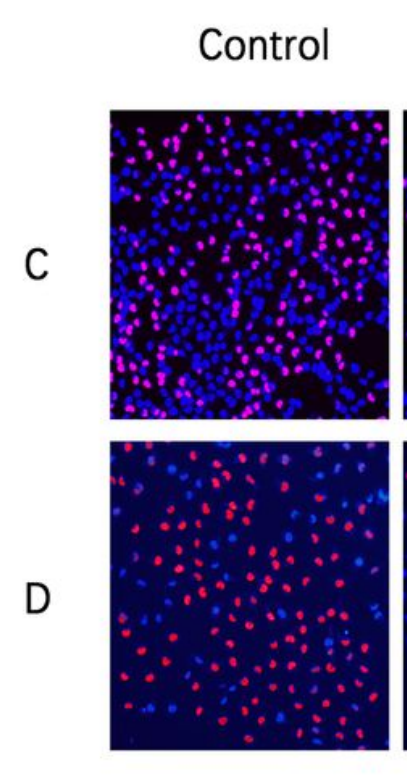

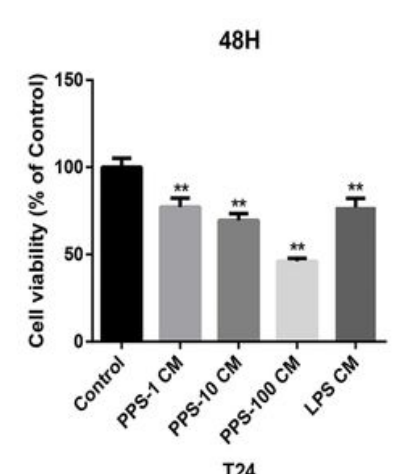

$\mathrm{T} 24$

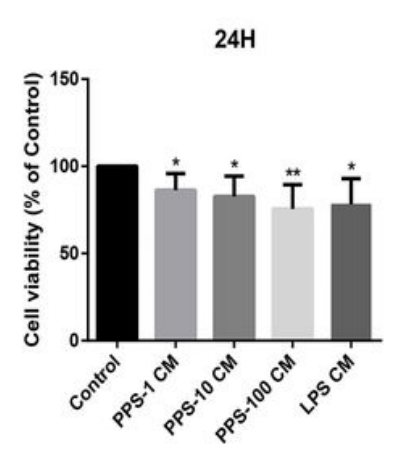

EJ

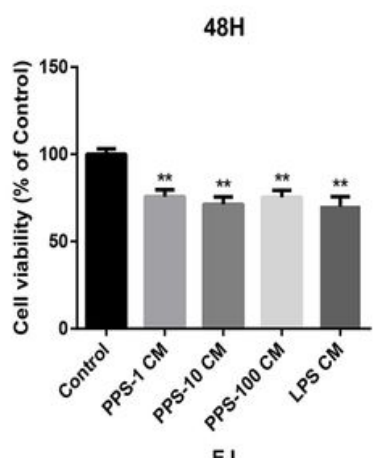

B
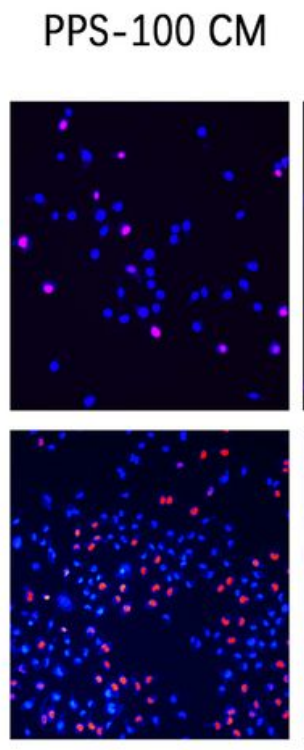

LPS CM
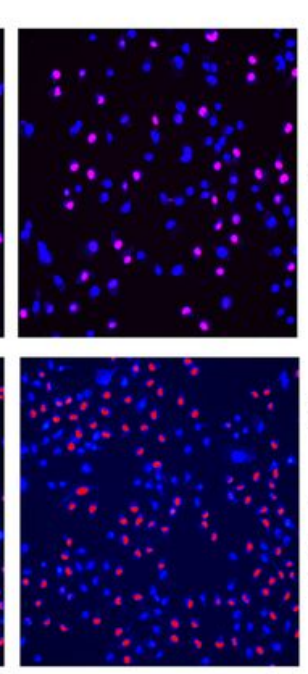
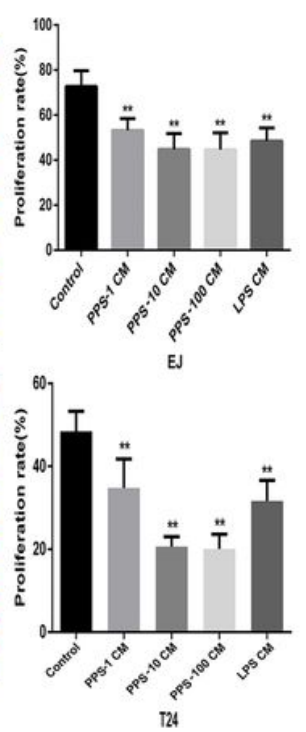

\section{Figure 3}

Cell viability and proliferation were restrained when incubated with the aforementioned supernatant. (A and B) Change in the cell viability (\% of the control group) when human bladder cancer cells T24 and EJ were cultured with the supernatant for $24 \mathrm{~h}$ or $48 \mathrm{~h}$. ( $\mathrm{C}$ and D) Effect of activated macrophages on T24 and EJ cell proliferation observed using EdU staining. Red fluorescence denotes EdU staining, representing proliferating cells. Blue fluorescence denotes DAPI staining, representing the staining of nuclei. Statistical data analysis showed a downward trend in cell proliferation. ${ }^{\star} P<0.05$; ${ }^{\star \star} P<0.01$ compared with the control group $(\mathrm{n}=3)$. 

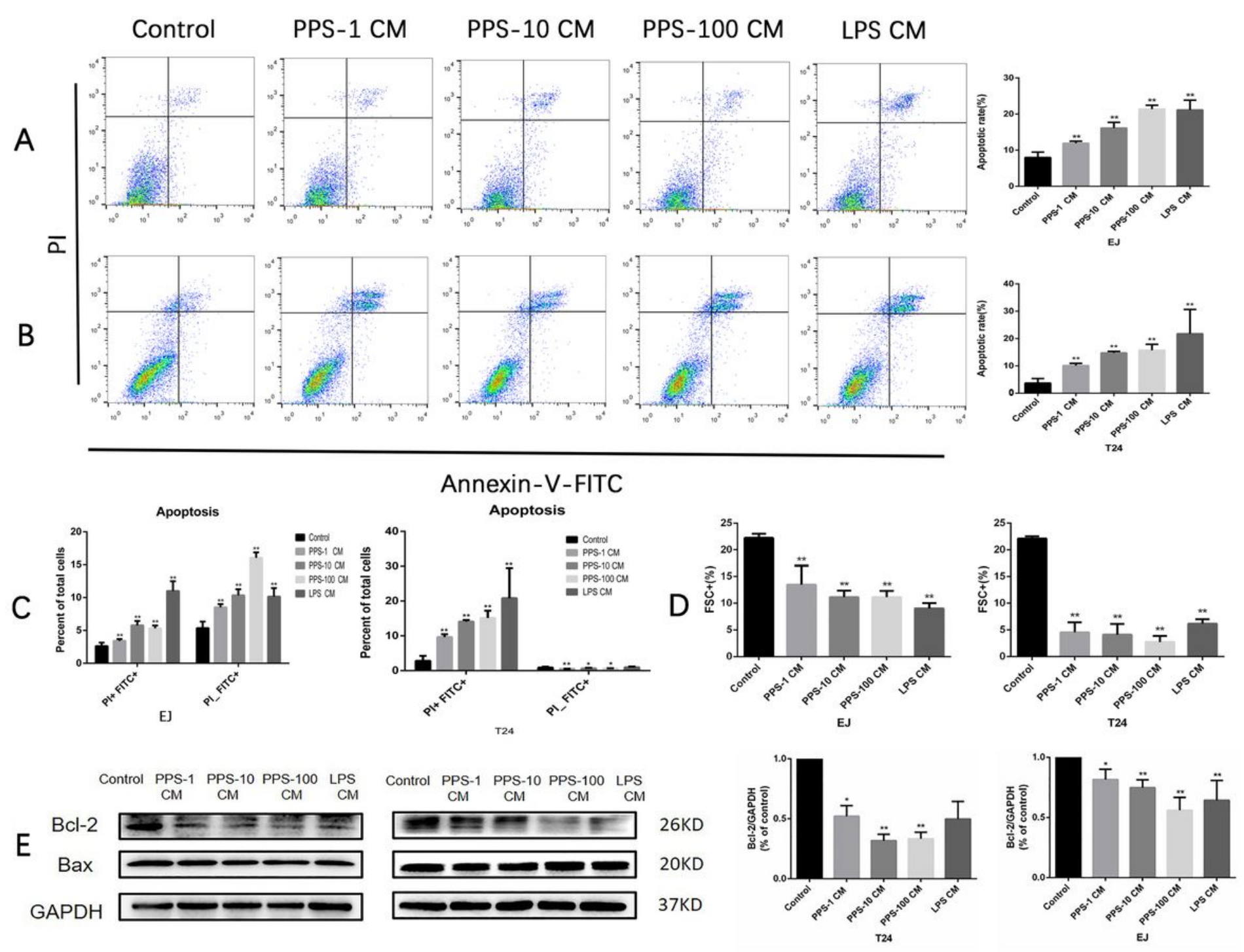

\section{Figure 4}

Detection of apoptosis by Annexin V/PI staining. (A and B) PPS-polarized macrophages facilitated the apoptosis of bladder cancer compared with the control group. (C) Statistical analysis showed the changes in early and late apoptosis of bladder cancer cell lines T24 and EJ in the co-culture system compared with the control group. (D) Experimental group showed decreased cell volume using flow cytometry. (E) Western blot analysis for apoptosis-related proteins Bcl-2 and BAX. The expression of GAPDH was used as a loading control. These experiments were repeated three times for statistics. ${ }^{*} \mathrm{P}<$ $0.05,{ }^{\star *} \mathrm{P}<0.01$ compared with the control group $(\mathrm{n}=3)$. 


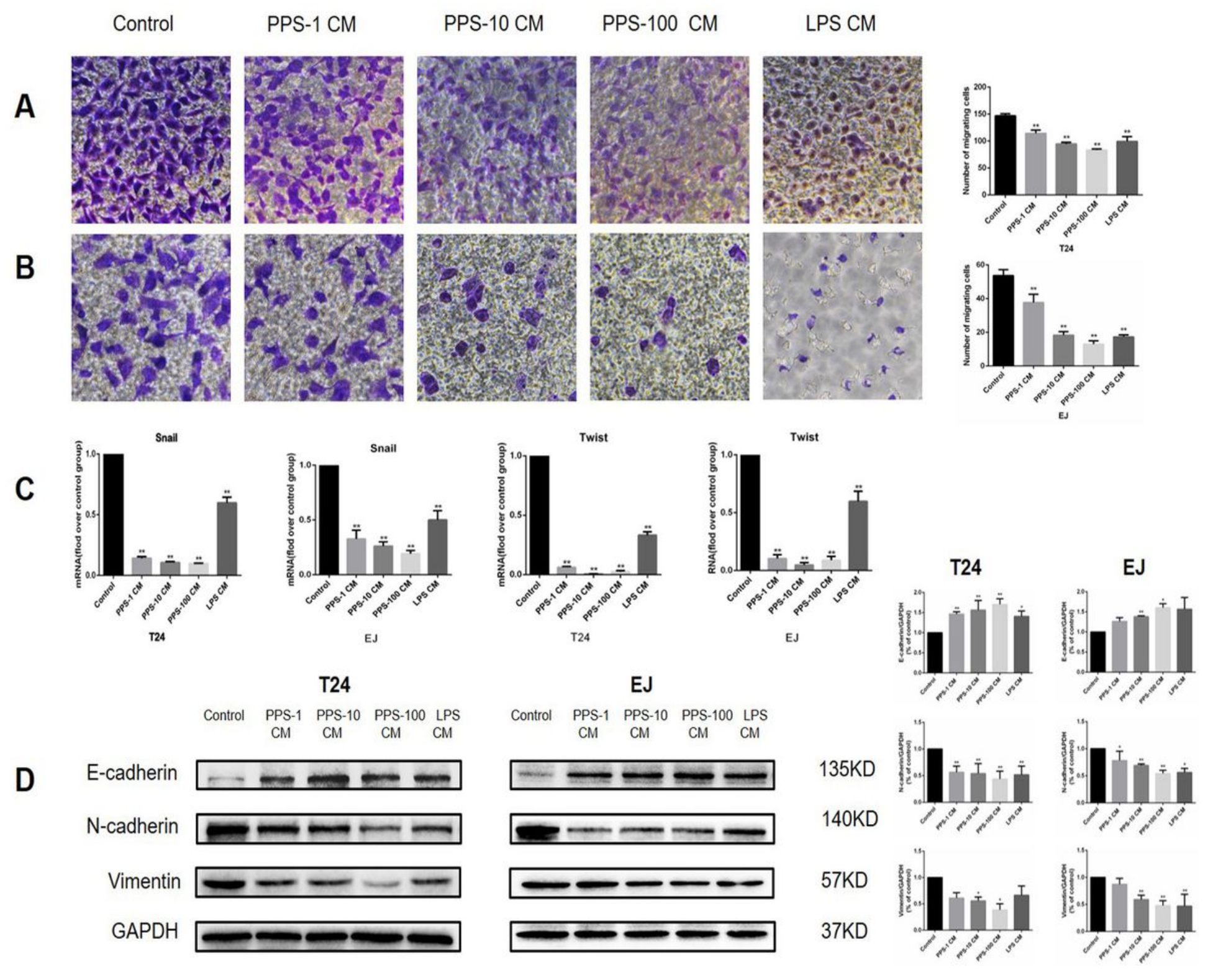

Figure 5

Determination of cell migration ability and epithelial-mesenchymal transition-related proteins using Transwell chamber and Western blot analysis. (A and B) Microscopic photography of cells penetrating the upper chamber and statistical analysis of migrating cells in different treatment groups (400x). (C) RTPCR analysis of expression of EMT transcription factors. (D) Western blot analysis of the expression of EMT marker proteins. ${ }^{*} \mathrm{P}<0.05,{ }^{*} \mathrm{P}<0.01$ compared with the control group $(\mathrm{n}=3)$. 
T24 Control PPS-1 PPS-10 PPS-100 LPS $\mathrm{CM} \quad \mathrm{CM} \quad \mathrm{CM} \quad \mathrm{CM}$

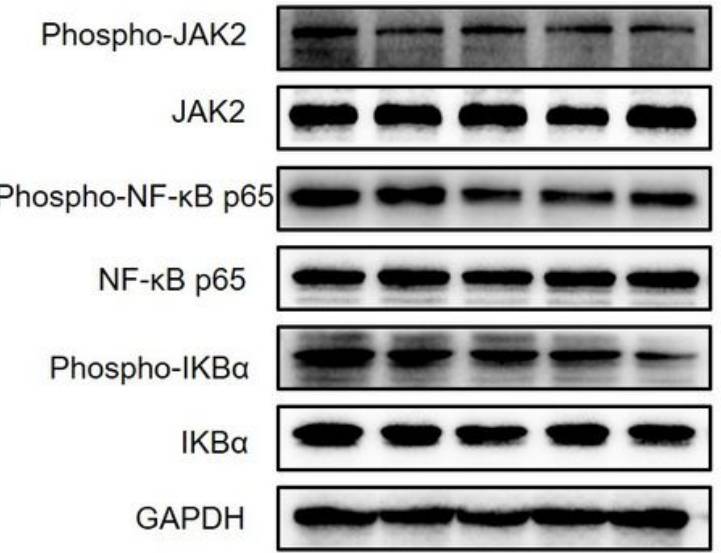

EJ

Control PPS-1 PPS-10 PPS-100 LPS

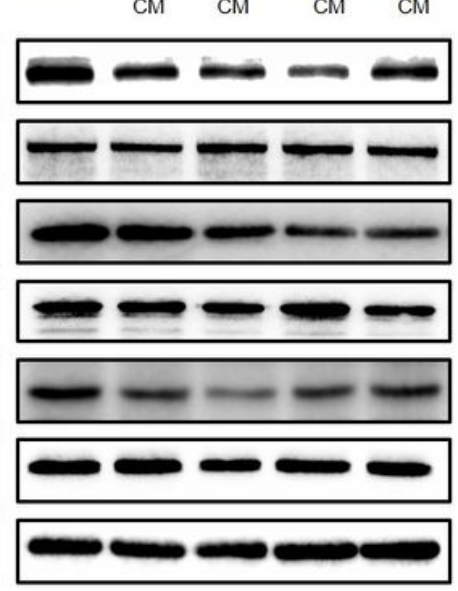

$125 \mathrm{KD}$

125KD

65KD

$65 K D$

$40 K D$

$39 K D$

37KD
T24

EJ
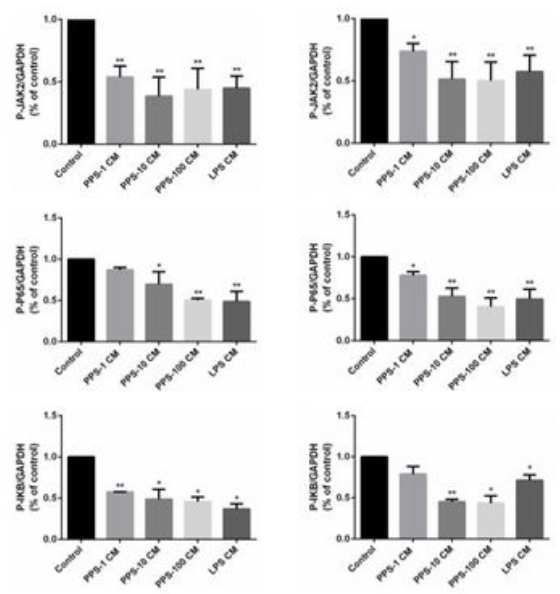

\section{Figure 6}

Detection of the expression of JAK2/NF- $\mathrm{B}$ pathway protein by Western blot analysis. The protein levels of p-JAK2, p-P65, and p-IKB were downregulated in bladder cancer cells T24 and EJ after culture with the conditioned medium. ${ }^{*} P<0.05,{ }^{*} \mathrm{P}<0.01$ compared with the control group $(\mathrm{n}=3)$.

\section{Supplementary Files}

This is a list of supplementary files associated with this preprint. Click to download.

- CERTIFICATEOFENGLISHEDITING.pdf 